\title{
Preparation and in Vitro Drug Release Evaluation of Once-Daily Metformin Hydrochloride Sustained-Release Tablets
}

\author{
Ling Zhao ${ }^{1}$, Yumeng Wei ${ }^{1 *}$, Yong Mei $^{2}$, Li Yang ${ }^{2}$, Yuan You ${ }^{2}$, Xufeng Yang ${ }^{2}$, Yanhong Jiang ${ }^{2}$ \\ ${ }^{1}$ Department of Pharmaceutics, School of Pharmacy, Luzhou Medical College, Luzhou, China; ${ }^{2}$ Chongqing Conquor Pharmaceutical \\ Co., Chongqing, China. \\ Email: ${ }^{*}$ zhaoling2006998@yahoo.com.cn
}

Received June $24^{\text {th }}, 2012$; revised July $25^{\text {th }}, 2012$; accepted August $11^{\text {th }}, 2012$

\begin{abstract}
The objective of this study was to develop once-daily metformin hydrochloride sustained-release tablets (MHSRT) and evaluate their in vitro release behavior. MHSRT were prepared by the film coating method. The in vitro drug release rate of MHSRT and the commercial tablets Fortamet ${ }^{\circledR}$ made in the United States of America in water was fitted with zero order kinetic equation, and Ritger-Peppas kinetic equation in $0.1 \mathrm{M} \mathrm{HCl}$ and $\mathrm{pH}$ 6.8-phosphate buffer, respectively. The similarity factor $f_{2}$ values of MHSRT in three different dissolution medium were 82,80 and 74, respectively in comparison with imported Fortamet ${ }^{\circledR}$, which were all greater than 50 . The results of storage-stability showed that MHSRT were stable for at least 6 months under stress condition $\left(40^{\circ} \mathrm{C} \pm 2^{\circ} \mathrm{C}, \mathrm{RH} 75 \% \pm 5 \%\right)$. Therefore, in this study, MHSRT were successfully prepared using optimized formulation technologies that meet mass produce. The in vitro release behavior of MHSRT was almost similar to that of imported Fortamet ${ }^{\mathbb{B}}$.
\end{abstract}

Keywords: Sustained-Release Tablets; Metformin Hydrochloride; In Vitro Release Rate; Similarity Factor; Kinetic Model

\section{Introduction}

Metformin hydrochloride (MH) is a biguanide oral antidiabetic drug which is widely used to treat non-insulin dependent diabetes mellitus (type 2 diabets). Its mode of action is thought to be multifactoral and includes delayed uptake of glucose from the intestinal tract, increased peripheral glucose utilisation mediated by increased insulin sensitivity and decreased hepatic and renal gluconeogenesis. In clinical treatment, there are many advantages of MH such as the tendency to weight reduction and the ability to reduce blood glucose to normal level without significant hypoglycaemia [1]. However, because of its relative low bioavailability $(40 \%-60 \%)$ and short biological half-life $(0.9-2.6 \mathrm{~h})$ [2-5], the immediate release dosage forms of $\mathrm{MH}$ such as conventional tablets and capsules, have to be administered three times a day [3], which results in a significant fluctuation in the plasma drug concentration and poor patient compliance. In order to overcome these problems, sustainedrelease drug delivery systems of MH including sustainedrelease matrix tablets [2,6-8], sustained-release pellets [3,9], sustained-release microparticles [10], prolonged

${ }^{*}$ Corresponding author. release microspheres [11], gastroretentive drug delivery preparation [12], $\mathrm{pH}$-controlled peroral delivery formulation [13] have been developed in recent years. Though sustained release formulations of $\mathrm{MH}$ have been reported to prolong drug release, formulation and technologies used in these studies were complicated and costly, which influence industrial scale and market expansion. At present, MHSRT produced by Bristol-Myers Squibb Company (USA) capture a major market share in China. However, many patients in our developing country could not accept it due to its high cost. Therefore, development of sustained release dosage form of $\mathrm{MH}$ that is similar to imported drug is to save pharmacy cost and improve clinical outcomes.

Therefore, in this study, MHSRT were prepared using optimized formulation technologies that meet mass produce. The in vitro drug release behavior of MHSRT was studied in water, $0.1 \mathrm{M} \mathrm{HCl}$ and $\mathrm{pH}$ 6.8-phosphate buffer as release medium and compared with the commercial tablets Fortamet ${ }^{\circledR}$ made in the United States of America.

\section{Materials and Methods}

\subsection{Materials}

Metformin hydrochloride (purity: 99.86\%) was purchased 
from Huainan Jiameng Pharmaceutical Co., Ltd (China). Carboxymethyl cellulose sodium (CMC) and pregelatinized Starch was obtained from Anhui Shanhe Pharmaceutical Excipient Co., Ltd (China). Ethyl cellulose was purchased from Shandong Heda Co., Ltd (China). PEG6000 was supplied by Liaoyang Aoke Nano Meterial Co., Ltd. (China). Hexadecanol was purchased from Hunan Erkang Pharmaceutical Co., Ltd (China). Magnesium stearate was purchased from Chongqing Chemical Reagent Co. Ltd. (China). Fortamet ${ }^{\mathbb{R}}$ (batch number: 4602094; expiry date: 20121102) were obtained from Watson Laboratories-Florida Ft. Lauderdale, FL 33314 (USA). were obtained from the United States of America. All chemicals and reagents used were of analytical grade. Water used in this study was double distilled water.

\subsection{Preparation of MHSRT}

Firstly, MH core tablets were prepared by wet granulation method. $\mathrm{MH}$ and $\mathrm{CMC}$ were mixed and the mixture was passed through a 100 -mesh sieve. Granulation was done using water. The wet mass was passed through fourteen meshes using the pendular granulator and the wet granules were air dried for about $2 \mathrm{~h}$. The granules were then sized by fourteen mesh sieve and mixed with pregelatinized starch and magnesium stearate. The core tablets were compressed on a tablet compression machine equipped with $12 \mathrm{~mm}$ convex punches. Secondly, the film coat suspension was prepared by dissolving and dispersing EC, PEG-6000 and hexadecanol in $95 \%$ ethanol. In brief, the above core tablets were placed into a fluid-bed spray coater and prewarmed to $35^{\circ} \mathrm{C}-40^{\circ} \mathrm{C}$ for $3-5 \mathrm{~min}$. The coating solution was delivered using peristaltic pump with a flow rate of $1.8 \mathrm{~mL} / \mathrm{min}$. Coating was carried out at $50^{\circ} \mathrm{C}$ inlet air temperature and $45^{\circ} \mathrm{C}$ outlet air temperature. The resulted MHSRT were dried at $40^{\circ} \mathrm{C}$ for $12 \mathrm{~h}$ and then were further performed quality evaluation.

\subsection{Determination of Drug Content}

According to the Chinese Pharmacopoeia (2010 version) about the determination of MH content, the samples (20 tablets) were taken and ground to fine powder. Then about $20 \mathrm{mg}$ powder was accurately weighed and placed in a $100 \mathrm{~mL}$ volumetric flask containing $75 \mathrm{~mL}$ water. After it was dissolved using ultrasonication at room temperature for $20 \mathrm{~min}$, this solution was diluted with water to $100 \mathrm{~mL}$ and mixed well, then filtered through a $0.45 \mu \mathrm{m}$ hydrophilic membrane. $2.5 \mathrm{~mL}$ of the solution was accurately taken and transferred to a $100 \mathrm{~mL}$ volumetric flask, and then was diluted with water to $100 \mathrm{~mL}$ and mixed well. The drug content was measured using an UV-visible spectrophotometer at a wavelength of
$233 \mathrm{~nm}$.

\subsection{Weight Variation Test}

To study weight variation, 20 tablets of each batch samples were weighed using an electronic balance (BT214D, Germany Sartorius), and the test was carried out according to the Chinese Pharmacopoeia (2010 version) method.

\subsection{Hardness and Friability Test}

For each batch of MHSRT, the hardness and friability of 12 tablets was determined using tablet with four measuring instrument (78X-6A).

\subsection{In Vitro Release Test}

In vitro release studies of MHSRT was carried out by the rotating basket methods of Chinese Pharmacopoeia (2010 version) appendix XD No.1. Six tablets of each batch of MHSRT were taken and placed in rotating basket, respectively. Then the rotating basket was introduced into $900 \mathrm{~mL}$ of each dissolution medium (water, $0.1 \mathrm{M}$ $\mathrm{HCl}$ and $\mathrm{pH} 6.8$ phosphate buffer) at $37^{\circ} \mathrm{C} \pm 0.5^{\circ} \mathrm{C}$ with a rotation speed of $100 \mathrm{rpm} .5 \mathrm{~mL}$ of sample solution was collected at different time intervals $(2,4,6,8,10,12 \mathrm{~h})$ and filtered through a $0.45 \mu \mathrm{m}$ hydrophilic membrane. $1.0 \mathrm{~mL}$ of subsequent filtrate was taken accurately to add into a $100 \mathrm{~mL}$ volumetric flask and diluted with the corresponding dissolution medium to $100 \mathrm{~mL}$ and mixed well. The amount of drug dissolved in the dissolution medium was measured using an UV-visible spectrophotometer at $233 \mathrm{~nm}$. The same volume of fresh dissolution medium at the same temperature was added to replace the amount withdrawn after each sampling.

The drug amount of cumulative release from the MHSRT was calculated with a standard curve prepared using bracketed concentration of $\mathrm{MH}$ each dissolution medium solution in a range from 15 to $125 \%$ of a theoretical concentration of $5.5 \mu \mathrm{g} / \mathrm{mL}$. The standard curve: $\mathrm{Y}=0.0746 \mathrm{X}-0.0031$ for distilled water; $\mathrm{Y}=0.0758 \mathrm{X}-$ 0.0034 for $0.1 \mathrm{M} \mathrm{HCl}$ and $\mathrm{Y}=0.0798 \mathrm{X}-0.0037$ for $\mathrm{pH}$ 6.8 phosphate buffer were obtained with coefficient of correlation $(r=0.9999)$.

\subsection{Data Analysis}

In order to evaluate the drug release kinetic model of the MHSRT, four kinetic models including the zero-order release equation, first-order release equation, Higuchi's equation and Ritger-Peppas ((1)-(4), respectively) were chosen to process the in vitro drug release data.

$$
\begin{gathered}
M t=k 1 t+b \\
\operatorname{Ln}(100-M t)=k 2 t+b
\end{gathered}
$$




$$
\begin{gathered}
M t=k 3 t^{1 / 2}+b \\
\operatorname{Ln} M t=k 4 \operatorname{Ln} t+b
\end{gathered}
$$

where $M t$ is the cumulative release percentage at time $t$, the $k 1, k 2, k 3$ and $k 4$ are the rate constant of the above kinetic equation, respectively.

In order to compare the difference of in vitro drug release behavior between the MHSRT, the similarity factor $f_{2}$ is used in this study and defined by the following equation (5).

$$
f_{2}=50 \times \log \left\{\left[1+(1 / n)^{2}\right]^{-0.5} \times 100\right\}
$$

where $n$ is the number of time point, $R t$ and $T t$ are the mean cumulative percentage drug dissolved at each time point, $t$. MHSRT developed in this study and imported Fortamet ${ }^{\circledR}$ made in the United States of America were chosen as test samples and reference preparation, respectively.

\subsection{Stability Test of MHSRT}

The accelerate stability testing was carried out according to the Technical Standard of Drug Stability Test of Chinese Pharmacopoeia (2010 version). The MHSRT samples were stored at $40^{\circ} \mathrm{C} \pm 2^{\circ} \mathrm{C}$, RH $75 \% \pm 5 \%$ for 6 months and the in vitro release was measured after 1, 2, 3 and 6 months of storage.

\section{Results and Discussion}

\subsection{Development of MHSRT}

In the preliminary study, MH, CMC, pregelatinized starch and magnesium stearate were chosen to prepare the core tablets as formulation composition. In addition, the film coating formulation was composed of EC, PEG-6000 and hexadecanol. On the basis of single factor experiments, we have confirmed the dosage range of the formulation composition. Then an orthogonal array was used to investigate the key influence on preparation. In the current research, the optimal formation of the core tablets was MH (500 g), CMC (40 g), pregelatinized starch $(30 \mathrm{~g})$ and magnesium stearate $(9 \mathrm{~g})$, the optimal formulation of the film coating was composed of EC (15 g), PEG-6000 (6 g) and hexadecanol (6 g) dissolved or dispersed in $95 \%$ ethanol resulting in $300 \mathrm{~mL}$ coating suspensions.
In order to validate whether the optimized formulation technologies of MHSRT can be suitable for mass produce, the three batches of MHSRT (100000 tablets/batch) were produced by Chongqing Conquor Pharmaceutical Co., Ltd. and the results were shown in Table 1 and Figures 1-3. Drug content was found to be uniform among the three batches of MHSRT and ranged from $97.85 \% \pm 0.09 \%$ to $99.36 \% \pm 0.07 \%$. The mean percentage weight variation of 20 tablets of each batch was less

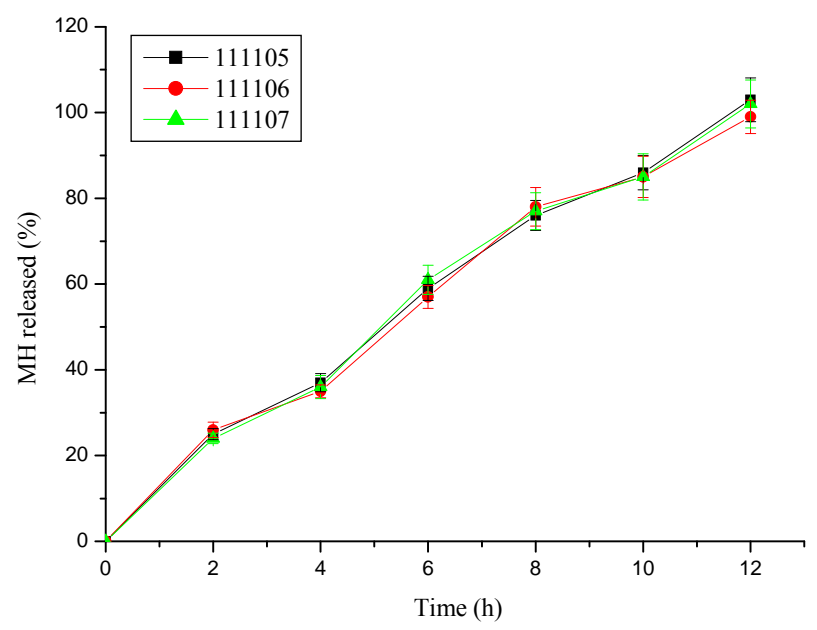

Figure 1. Drug release profiles of the three batches (111105, 111106, and 111107) of MHSRT in water as dissolution medium.

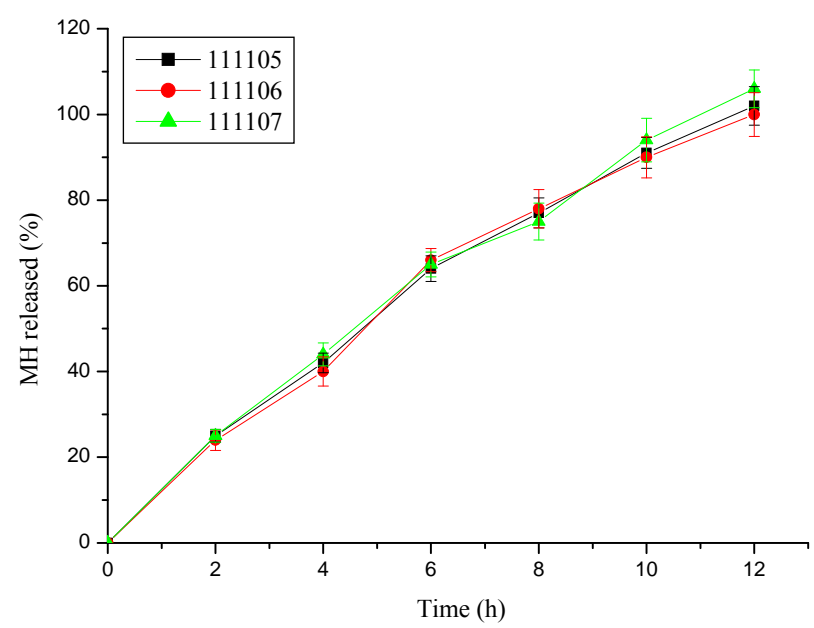

Figure 2. Drug release profiles of the three batches $(111105$, 111106, and 111107) of MHSRT in $0.1 \mathrm{M} \mathrm{HCl}$ as dissolution medium.

Table 1. Properties of the three batches of MHSRT prepared in this study.

\begin{tabular}{ccccc}
\hline Batch & Drug content $(\%)$ & Deviation in weight variation $(\%)$ & Hardness $\left(\mathrm{kg} / \mathrm{cm}^{2}\right)$ & Friability $(\%)$ \\
\hline 111105 & $98.55 \pm 0.05$ & $3.26 \pm 0.02$ & $6.8 \pm 0.22$ & $0.72 \pm 0.04$ \\
111106 & $99.36 \pm 0.07$ & $2.90 \pm 0.04$ & $6.1 \pm 0.17$ & $0.79 \pm 0.05$ \\
111107 & $97.85 \pm 0.09$ & $3.86 \pm 0.03$ & $7.4 \pm 0.26$ & $0.55 \pm 0.04$ \\
\hline
\end{tabular}


than $5.0 \%$. The hardness and percentage friability of the tablets of all batches ranged from $6.1 \pm 0.17 \mathrm{~kg} / \mathrm{cm}^{2}$ to $7.4 \pm 0.26 \mathrm{~kg} / \mathrm{cm}^{2}$ and $0.55 \% \pm 0.04 \%$ to $0.79 \% \pm 0.05 \%$, respectively. The in vitro drug release behaviors of the three batches of MHSRT in three different dissolution medium were almost similar. Therefore, the optimized formulation technologies possess good reproduction, which are suitable for mass produce.

\subsection{Evaluation of in Vitro Drug Release}

The dissolution profiles of MHSRT developed in this study and imported Fortamet ${ }^{\circledR}$ made in the United States of America in water, $0.1 \mathrm{M} \mathrm{HCl}$ and $\mathrm{pH} 6.8$ phosphate buffer as release medium are shown in Figure 4. It was found from Figure 4 that the release rate of MHSRT was similar to that of Fortamet ${ }^{\mathbb{R}}$. In the first $2 \mathrm{~h}$, the cumulative release percentages of MHSRT and Fortamet ${ }^{\circledR}$ were $25 \%$ and $27 \%$ in water; $25 \%$ and $22 \%$ in $0.1 \mathrm{M} \mathrm{HCl}$; $22 \%$ and $20 \%$ in pH 6.8 phosphate buffer, respectively. After $6 \mathrm{~h}$, the cumulative release percentages of MHSRT and Fortamet ${ }^{\circledR}$ were $59 \%$ and $56 \%$ in water; $64 \%$ and $62 \%$ in $0.1 \mathrm{M} \mathrm{HCl} ; 58 \%$ and $51 \%$ in $\mathrm{pH} 6.8$ phosphate buffer, respectively.

As we all know, the different release kinetic models are assumed to reflect different release mechanisms [14]. Therefore, in this study, zero-order release equation, first-order release equation, Higuchi's equation and Ritger-Peppas were used to analyze the in vitro released data. Correlation coefficients of Zero-order, First-order, Higuchi's equation and Ritger-Peppas kinetic models used in this study were shown in Tables 2-4. It can be seen that the in vitro drug release rate of MHSRT and Fortamet ${ }^{\circledR}$ in water showed a zero order approximately kinetic model and could be described by the following equation: $M t=7.915 t+8.934(\mathrm{R}=0.996)$ and $M t=$ $7.843 t+9.934(\mathrm{R}=0.996)$. However, the release rate of MHSRT and Fortamet ${ }^{\circledR}$ in $0.1 \mathrm{M} \mathrm{HCl}$ and $\mathrm{pH} 6.8$ phosphate buffer was fitted with Ritger-Peppas kinetic models

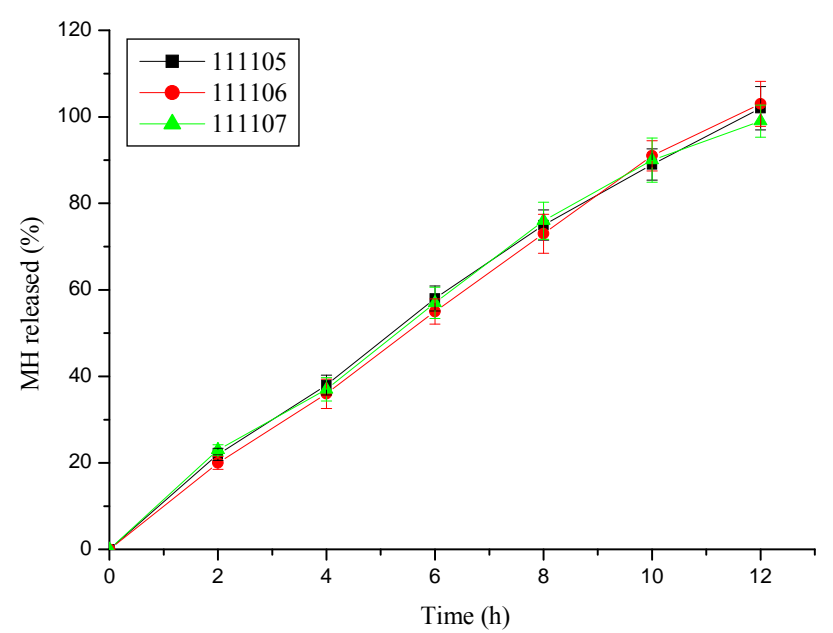

Figure 3. Drug release profiles of the three batches (111105, 111106, and 111107) of MHSRT in pH 6.8 phosphate buffer as dissolution medium.

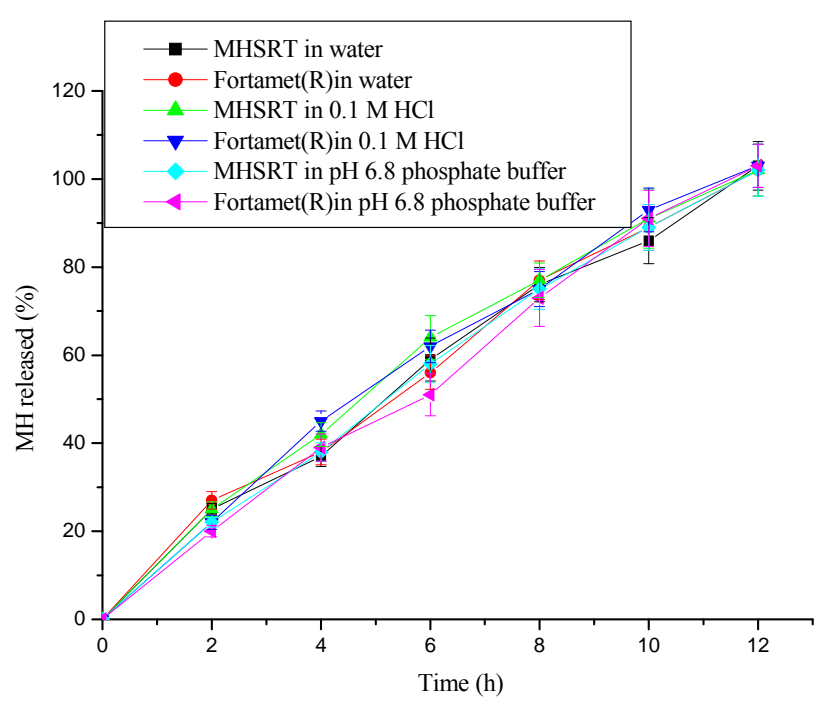

Figure 4. Drug release profiles of MHSRT developed in this study and imported Fortamet ${ }^{\circledR}$ in water, $0.1 \mathrm{M} \mathrm{HCl}$ and $\mathrm{pH}$ 6.8 phosphate buffer as dissolution medium.

Table 2. Correlation coefficients of kinetic models used for evaluate the in vitro release behavior of MHSRT and Fortamet ${ }^{\circledR}$ in water as dissolution medium.

\begin{tabular}{ccccc}
\hline Sample & Zero-order & First-order & Higuchi's equation & Ritger-peppas \\
\hline MHSRT & 0.996 & 0.893 & 0.991 & 0.993 \\
Fortamet $^{\circledR}$ & 0.996 & 0.910 & 0.989 & 0.991 \\
\hline
\end{tabular}

Table 3. Correlation coefficients of kinetic models used for evaluate the in vitro release behavior of MHSRT and Fortamet ${ }^{\circledR}$ in $0.1 \mathrm{M}$ HCl as dissolution medium.

\begin{tabular}{ccccc}
\hline Sample & Zero-order & First-order & Higuchi's equation & Ritger-peppas \\
\hline MHSRT & 0.987 & 0.853 & 0.996 & 0.998 \\
Fortamet $^{\circledR}$ & 0.988 & 0.865 & 0.993 & 0.997 \\
\hline
\end{tabular}


Table 4. Correlation coefficients of kinetic models used for evaluate the in vitro release behavior of MHSRT and Fortamet ${ }^{\circledR}$ in pH 6.8 phosphate buffer as dissolution medium.

\begin{tabular}{ccccc}
\hline Sample & Zero-order & First-order & Higuchi's equation & Ritger-peppas \\
\hline MHSRT & 0.995 & 0.827 & 0.993 & 0.999 \\
Fortamet $^{\circledR}$ & 0.994 & 0.834 & 0.982 & 0.996 \\
\hline
\end{tabular}

and could be described by the following equation: $\mathrm{LnMt}=$ $0.801 \mathrm{Ln} t+2.665(\mathrm{R}=0.998)$ and $\mathrm{LnMt}=0.857 \mathrm{Ln} t+$ $2.551(\mathrm{R}=0.997) ; \mathrm{LnMt}=0.875 \mathrm{Ln} t+2.471(\mathrm{R}=0.999)$ and $\mathrm{LnMt}=0.922 \mathrm{Ln} t+2.354(\mathrm{R}=0.996)$, respectively, which indicated that the drug release mechanisms of MHSRT and Fortamet ${ }^{\circledR}$ were similar in gastrointestinal tract. According the formulation composition MHSRT, it was obvious that drug diffusion and erosion were the main factor in controlling the drug release rate from MHSRT. This was also evidenced by the value of the release exponent $n$ of 0.801 and 0.875 , because when $n$ ranged from 0.45 to 0.89 , indicating that drug is released by the combination of diffusion and erosion mechanisms [15-16].

On the other hand, the similarity factor $f_{2}$ value between 50 and 100 shows that two release profiles are similar [17]. Therefore, in order to evaluate the in vitro release difference of MHSRT, when imported Fortamet ${ }^{\circledR}$ were chosen as reference, $f_{2}$ value was calculated. It was found that the dissolution profile of only MHSRT prepared in this study was similar to that of Fortamet ${ }^{\circledR}$, because the $f_{2}$ values in water, $0.1 \mathrm{M} \mathrm{HCl}$ and $\mathrm{pH} 6.8$ phosphate buffer were 82,80 and 74 , respectively, which were all greater than 50 .

\subsection{Test for Stability}

It was known that the stability of preparation is an important factor to estimate the quality of pharmaceutical formulation. Thus, the acceleration stability test was performed to study the stability of MHSRT. It can be seen from Figures 5-7 that good storage stability was observed and the in vitro release profiles had little change. And no significant difference in cumulative release percentage of drug in water, $0.1 \mathrm{M} \mathrm{HCl}$ and $\mathrm{pH} 6.8$ phosphate buffer after 1, 2, 3 and 6 months was observed in comparison with MHSRT samples before storage $(n=3$; $P>0.05)$. Therefore, MHSRT developed in this study were stable at least for 6 months under stress conditions.

\section{Conclusion}

In this study, once-daily metformin hydrochloride sustained-release tables (MHSRT) were successfully developed by the optimized formulation technologies that are suitable for mass produce at Chongqing Conquor Pharmaceutical Co., Ltd. The in vitro release behavior of MHSRT was almost similar to that of imported For-

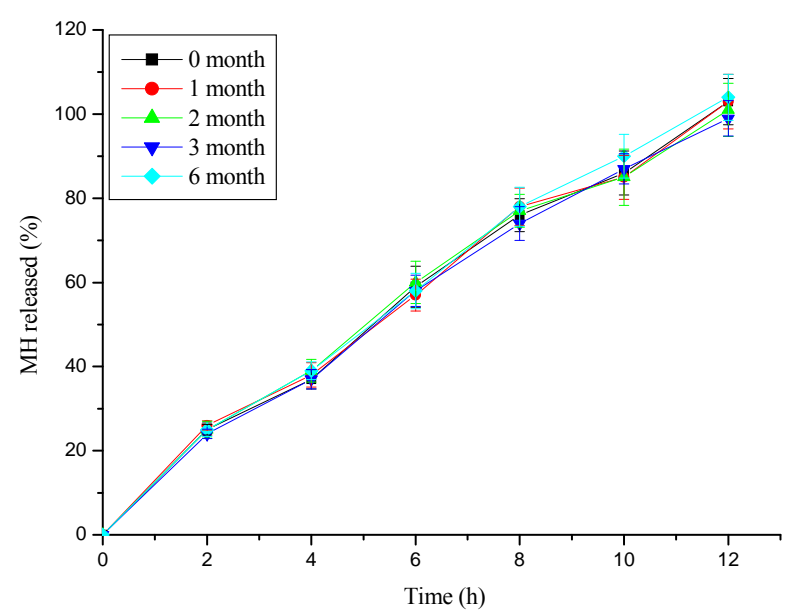

Figure 5. Drug release profiles of MHSRT developed in this study in water before and after 1, 2, 3 and 6 months of storage under stress conditions $\left(40^{\circ} \mathrm{C} \pm 2^{\circ} \mathrm{C}\right.$, $\mathrm{RH} 75 \% \pm$ $5 \%)$.

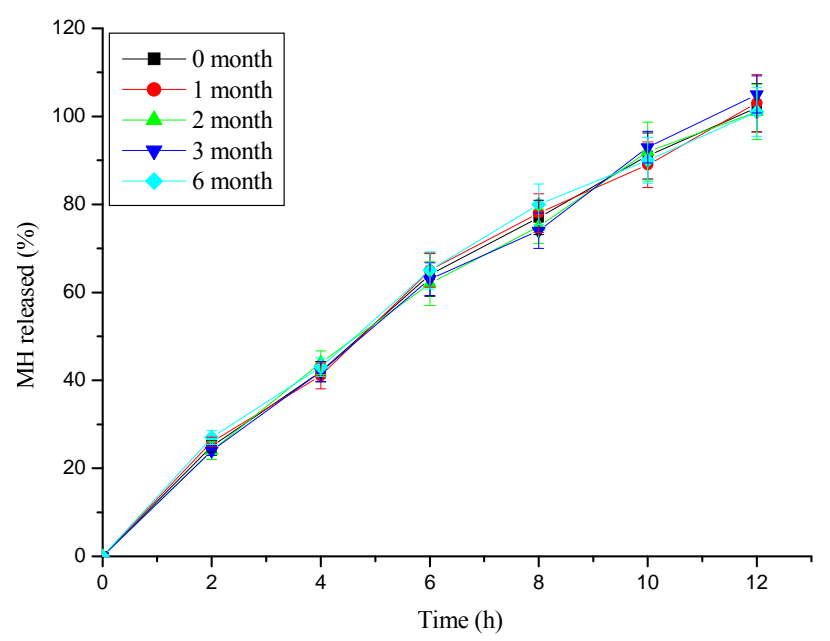

Figure 6. Drug release profiles of MHSRT developed in this study in $0.1 \mathrm{M}$ HCl before and after 1, 2, 3 and 6 months of storage under stress conditions $\left(40^{\circ} \mathrm{C} \pm 2^{\circ} \mathrm{C}, \mathrm{RH} 75 \% \pm\right.$ $5 \%)$.

tamet $^{\circledR}$. Furthermore, MHSRT developed in this study were stable at least for 6 months under stress conditions.

\section{Acknowledgements}

This research was supported of by the National Natural Science Foundation of China (81101678), the Key Program of the Scientific Research Foundation of the Edu- 


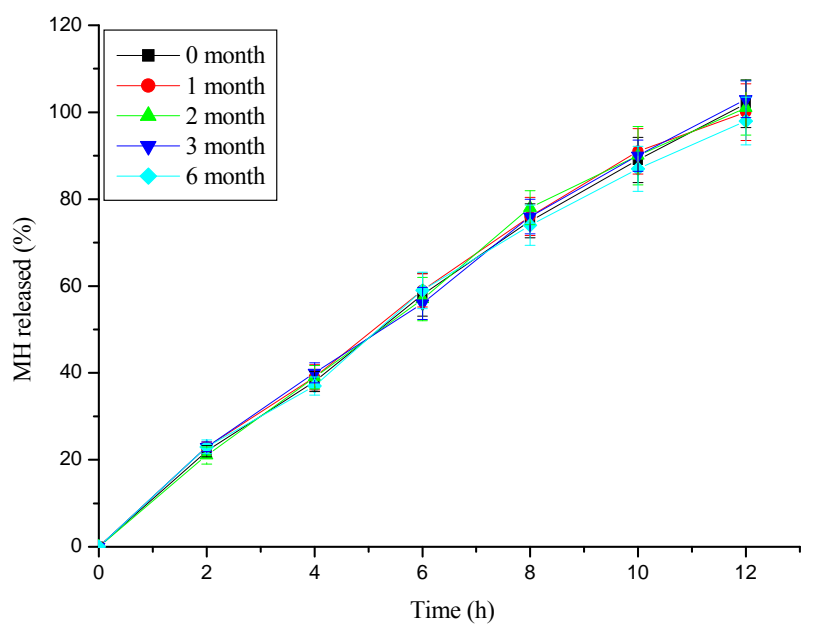

Figure 7. Drug release profiles of MHSRT developed in this study in pH 6.8 phosphate buffer before and after $1,2,3$ and 6 months of storage under stress conditions $\left(40^{\circ} \mathrm{C} \pm 2^{\circ} \mathrm{C}\right.$, RH $75 \% \pm 5 \%)$.

cation Department of Sichuan Province [09ZA049; 11ZZ024], the Scientific Research Foundation of the Health Bureau of Sichuan Province [100215; 100214]and the Key Program of the Scientific Research Foundation of Bureau of Science and Technology of luzhou Municipality [2011-S-32(1/4)].

\section{REFERENCES}

[1] X.-X. Yin, Y.-D. Zhang, H.-T. Zhuo, J.-P. Shen, L.-M. Li and Q. Jun, "Pharmacokinetics and Relative Bioavailability of Metformin Hydrochloride Sustained Release Tablets in Healthy Chinese Volunteers," Asian Journal of Drug Metabolism and Pharmacokinetics, Vol. 4, No. 2, 2004, pp.137-142.

[2] G. Corti, M. Cirri, F. Maestrelli, N. Mennini and P. Mura, "Sustained-Release Matrix Tablets of Metformin Hydrochloride in Combination with Triacetyl-Beta-Cyclodextrin," European Journal of Pharmaceutics and Biopharmaceutics, Vol. 68, No. 2, 2008, pp. 303-309. doi:10.1016/j.ejpb.2007.06.004

[3] L. D. Hu, Y. Liu, X. Tang and Q. Zhang, "Preparation and in Vitro/in Vivo Evaluation of Sustained-Release Metformin Hydrochloride Pellets," European Journal of Pharmaceutics and Biopharmaceutics, Vol. 64, No. 2, 2006, pp. 185-192. doi:10.1016/j.ejpb.2006.04.004

[4] P. J. Pentikäinen, P. J. Neuvonen and A. Penttilä, "Pharmacokinetics of Metformin after Intravenous and Oral Administration to Man," European Journal of Clinical Pharmacology, Vol. 16, No. 3, 1979, pp. 195-202. doi:10.1007/BF00562061

[5] G. T. Tucker, C. Casey, P. J. Phillips, H. Connor, J. D. Ward and H. F. Woods, "Metformin Kinetics in Healthy Subjects and in Patients with Diabetes Mellitus," British Journal of Clinical Pharmacology, Vol. 12, No. 2, 1981, pp. 235-246.

[6] P. K. Bhoyar and D. M. Biyani, "Formulation and in Vitro
Evaluation of Sustained Release Dosage Form with Taste Masking of Metformin Hydrochloride," Indian Journal of Pharmaceutical Sciences, Vol. 72, No. 2, 2010, pp. 184190. doi:10.4103/0250-474X.65031

[7] O. Defang, N. Shufang, L. Wei, G. Hong, L. Hui and P. Weisan, "In Vitro and in Vivo Evaluation of Two Extended Release Preparations of Combination Metformin and Glipizide," Drug Development and Industrial Pharmacy, Vol. 31, No. 7, 2005, pp. 677-685. doi: $10.1080 / 03639040500216410$

[8] A. Ghosh, A. Bose and U. Bhaumik, "Application of Response Surface Methodology in the Formulation of Sustained Release Matrix Tablets of Metformin Hydrochloride," Asian Journal of Chemistry, Vol. 20, No. 7, 2008, pp. 5541-5556.

[9] J. Piao, J. E. Lee, K. Y. Weon, D. W. Kim, J. S. Lee, J. D. Park, Y. Nishiyama, I. Fukui and J. S. Kim, "Development of Novel Mucoadhesive Pellets of Metformin Hydrochloride," Archives of Pharmacal Research, Vol. 32, No. 3, 2009, pp. 391-397. doi:10.1007/s12272-009-1312-0

[10] P. Subhedar, J. B. Naik and D. N. Muley, "Effect of Polymer Concentration on Sustained Release Microparticles of Metformin Hydrochloride Prepared by Using Spray Dryer," Polymer-Plastics Technology and Engineering, Vol. 49, No. 3, 2010, pp. 267-271.

[11] H. F. Liu, X. Y. Su, X. Li, X. Zhao, L. Zang and W. S. Pan, "Development of Prolonged Release Microspheres of Metformin Hydrochloride Using Ion Exchange Resins," Journal of Chinese Pharmaceutical Sciences, Vol. 15, No. 3, 2006, pp. 155-161.

[12] S. P. Boldhane and B. S. Kuchekar, "Gastroretentive Drug Delivery of Metformin Hydrochloride: Formulation and in Vitro Evaluation Using 32 Full Factorial Design," Current Drug Deliver, Vol. 6, No. 5, 2009, pp. 477-485.

[13] G. Di Colo, S. Falchi and Y. Zambito, "In Vitro Evaluation of a System for pH-Controlled Peroral Delivery of Metformin," Journal of Controlled Release, Vol. 80, No. $1-3,2002$, pp. 119-128. doi:10.1016/S0168-3659(02)00022-6

[14] P. C. Wu, M. J. Tsai, Y. B. Huang, J. S. Chang and Y. H. Tsai, "In Vitro and in Vivo Evaluation of Potassium Chloride Sustained Release Formulation Prepared with Saturated Polyglycolyed Glycerides Matrices," International Journal of Pharmaceutics, Vol. 243, No. 1-2, 2002, pp. 119-124. doi:10.1016/S0378-5173(02)00264-8

[15] J. E. Polli, G. S. Rekhi, L. L. Augsburger and V. P. Shah, "Methods to Compare Dissolution Profiles and a Rationale for Wide Dissolution Specifications for Metoprolol Tartrate Tablets," Journal of Pharmaceutical Sciences, Vol. 86, No. 6, 1997, pp. 690-700. doi:10.1021/js960473x

[16] S. G. Wang, L. Wang, Z. Z. Shao, X. P. Lu and X. Q. Hang, "Study on the Release Rate of Niacin in Different Media for the Compound Niacin Extended-Release and Simvastatin Tablets," Chinese Journal of Pharmacology and Toxicology, Vol. 8, No. 10, 2011, pp. 581-584.

[17] N. A. Peppas, "Analysis of Fickian and Non-Fickian Drug Release from Polymers," Pharmaceutica Acta Helvetiae, Vol. 60, No. 4, 1985, pp. 110-111. 